\title{
Radial as the Default Approach: Are We Overexaggerating?
}

\author{
Nicolaus Reifart* and James Margolis \\ Main Taunus Heart Institute, Bad Soden, Germany \\ ${ }^{\star}$ Corresponding author: Nicolaus Reifart, Main Taunus Heart Institute, Bad Soden, Germany; E-mail: reifart@gmx.com
}

Received: November 16, 2020; Accepted: November 25, 2020; Published: December 03, 2020

\begin{abstract}
Recent guidelines and reviews recommend trans radial approach (TRA) as a standard strategy for PCI unless there are overriding procedural recommendations [1-3]. The basis for these recommendations is randomised trials and metanalyses with conflicting results. We seek to unmask common misinterpretations in showing that the unfavourable results of TFA compared to TRA are most likely due to flawed study protocols with missing protocols for the TFA procedure and failure to correctly address vascular complications in the trans radial arm. Properly designed trials are mandatory before superseding TFA as a strategy that according to our combined experience of more than 25,000 coronary interventions should by no means be inferior to TRA yet with the tremendous advantage of ease of procedures, higher success-rates and less radiation.
\end{abstract}

\section{Introduction}

Trans radial access (TRA) has become the default access in Europe and Asia and its use is rapidly growing in Germany and the United States [4]. Enthusiasm for TRA has reached such a feverish pitch that criticism of TRA or praise for transfemoral access (TFA) is looked upon with disdain. With forty years' experience including significant radial and brachial experience and a personal caseload in excess of 25,000 procedures we fail to see relevant advantage in the radial procedure. Although we agree that in most cases trans radial is a reasonable alternative to transfemoral, we have reason to doubt sufficient evidence to justify a $1 \mathrm{~A}$ guideline recommendation ("standard approach") for TRA [1].

\section{Aim of the Review}

Major sources of evidence were the three recent large randomised trials (RIVAL, MATRIX and SAFARI-STEMI) as well as two recent metanalyses. We are convinced that arguing for radial as default and the increasing disregard of PCI via femoral route in current guidelines and large comparative trials [5-8] needs to be challenged since it requires first and foremost more properly designed studies.

\section{General Aspects}

Prior to an analysis of the data it is important to understand that generally practiced techniques differ significantly from optimal techniques, and this is the case for both femoral and radial access. Large scale clinical trials include a spectrum of operators from highly skilled to "just learning" and none of the trials specify proper technique for either TRA or TFA. Nor do they report any uniformity of technique with either procedure. In light of the small differences and marginal $p$ values between the TRA and TFA groups, differences in technique, especially with TFA would be sufficient to negate the findings. It is agreed that as practiced in Europe and the US, excess major bleeding associated with TFA is a problem, especially in acute coronary syndromes and possibly in elective PCI. We also agree that there may be a slight increase in all-cause mortality with TFA vs. TRA, and this difference is most likely due to bleeding complications. But we are also convinced that there would be no difference upon proper application of transfemoral approach (see appendix). However, unless there is a new trial with improved techniques included in the protocol, our persuasion remains speculative.

Bleeding aside, there are many advantages to TFA and many disadvantages to TRA. None of the randomised studies and metanalyses that demonstrate excess bleeding and higher mortality with TFA address these other factors. Properly designed randomized studies must limit themselves to one or two primary endpoints and a few secondary endpoints that can be used to generate hypotheses for future studies. Designers of clinical studies must guess at which parameters need to be evaluated to assure that the results are relevant and useful. When comparing TRA and TFA we need to look at much more than the small differences in complications and the barely demonstrable differences in mortality. TFA is technically easier. In the RIVAL trial, $7 \%$ of patients randomized to TRA had to switch to TFA because of difficulties with radial artery [9].

On the other hand, we have no doubt that TFA will result in more access site bleeding complications if not complied with proper technique of puncturing and sheath removal. In addition immediate ambulation after interventions is only possible following closure device (e.g. Angio-Seal ${ }^{\circ}$ ) [10] While most radial patients may be ambulated immediately, driving cost savings [11]. TRA is technically more difficult with lower success rates for coronary angiography and PCI $[9,12]$, results in poorer image quality and increased radiation exposure to operators and patients $[13,14]$ Radial artery closure might 
occur in 5-10\%, but is generally considered benign if the ulnar artery is intact $[15,16]$. However due to potential endothelial damage after coronary angiography, use of the radial artery as conduit for CABG is discouraged [17]. Cath labs that use TRA as their "default" technique are less likely to properly train younger physicians in TFA. More importantly, these labs will not develop the culture that is required to prevent major bleeding in TFA cases.

\section{Bleeding and Vascular Complications}

Bleeding complications after PCI, related to the vascular access site, may increase the risk of periprocedural mortality by $1.3-2.1 \%$ [18]. In a large registry of 335,477 patients who underwent PCI in 2002 a major vascular complication occurred in $4.85 \%$ [19]. With experience however the risk of any vascular complication can be decreased from $1.7 \%$ for diagnostic procedures and $3.1 \%$ for PCI to very low numbers of $0.2 \%$ and $1.0 \%$ respectively [20], a finding that is confirmed in a large scale monitor-controlled registry reporting bleeding complications prolonging hospital stay, or requiring blood transfusion or surgical intervention, being as low as $<0.1 \%$ following diagnostic procedures (mainly with $4 \mathrm{~F}$ ) and about $1 \%$ following PCI of ACS [21]. As for RIVAL (published in 2011), the rate of nonCABG-related major bleeding at 30 days was $0.7 \%$ in the radial group compared with $0.9 \%$ in the femoral group and only $0.2 \%$ and $0.3 \%$ of the bleeding events could be attributed to the access site [9]. In the MATRIX trial (published in 2015) bleeding complications (BARC $3-5)$ occurred in only $0.8 \%$ of the transfemoral patients and TIMI major or minor bleeding in $1.2 \%$ (TRA) and $1.7 \%$ (TFA) ( $\mathrm{p}=0.08$ ). In the SAFARI-STEMI randomized trial, published in 2020, bleeding complications ware rare and did not differ ( $1.4 \%$ vs. $2.0 \%$; RR, 0.71$)$ [22].

Thus, contemporary bleeding complications after transfemoral approach in ACS-PCI are in the range of 1-2/100 and might not be different from TRA in the hands of experienced TFA operators. We strongly believe that it is possible to meaningfully decrease access site bleeding with more careful attention to technique with the femoral puncture and sheath removal. Liberal use of vascular closure devices has been shown to reduce bleeding by $50 \%$ [23], and therefore should be offered at least all high risk patients - e.g., obese patients, patients with severe hypertension, and those who have received anticoagulation as part of their procedure. It is our practice to use vascular closure devices on virtually all post PCI patients. If the access site complication rates for TFA in the general community can be reduced by $50 \%$, the arguments favouring TRA become superficial. Endothelial damage occurs in all patients after trans radial coronary angiography [17], and radial artery occlusion after trans radial intervention, a mostly quiescent complication, is likely to occur in $5-8 \%$ of the patients, relative to timing of assessment $(7.7 \%$ after 24 hours and $5.5 \%$ at $>1$ week follow-up [24]. It is often overlooked because $>50 \%$ of operators do not even assess radial artery patency before discharge [25] and it is not even mentioned in one randomised trial nor in metanalyses. It is by no means a trivial complication because future use of the radial artery as access, conduit for bypass-surgery or fistula formation in haemodialysis patients is precluded.

\section{Major Adverse Events}

Major adverse events, defined as $30 \mathrm{~d}$ composite of all-cause mortality, myocardial infarction, or stroke occur in about $6-10 \%$ of patients who receive PCI due to ACS. In many studies comparing TRA to TFA all-cause mortality was increased in the TFA group $[8,26]$. In most studies the increase did not reach statistical significance, mortality was not a prespecified endpoint, and in none was it shown to persist after controlling for bleeding. As shown by a pairwise and network meta-analysis of randomized controlled trials, the survival differences appeared patient-related and not driven by beneficial effects of TRA [27]. RIVAL, the first of the two large randomised trials, failed to show any difference in the primary endpoint (MI/stroke/ severe bleeding $3.2 \%$ vs. $4 \% ; \mathrm{p}=0.5$ ) or secondary endpoint (death/ $\mathrm{MI} /$ stroke $3.2 \%$ vs. $3.2 \%$ ) [9].

With MATRIX, the second randomised comparison, there was no significant difference between radial access and femoral access in terms of the first co-primary endpoint of 30day MACE, (RR 0.85, 95\% CI 0.74-0.99, two sided $\mathrm{P}=0.031$; non-significant at a pre-specified $\alpha$ of 0.025 ). All-cause mortality was $1.6 \%$ and $2.2 \%$ respectively ( $\mathrm{p}=$ 0.045 ) but this was not a pre-specified endpoint [12]. The results of MATRIX must be interpreted with caution due to a strong modulating effect of operator/center experience on the efficacy [28] because the benefit of radial over femoral access obviously depends upon the operator's expertise in the femoral technique: The MATRIX study divided patients into 3 groups based on the participating center's proportion of radial PCIs: "low" (14.9\% to 64.4\%), intermediate ( $65.4 \%$ to $79.0 \%)$, and high $(80.0 \%$ to $98.0 \%)$. The results of this stratified analysis showed that the centers with low and intermediate experience in radial approach had similar MACE rates (between 7.5\% and $8.5 \%$ both for radial as well as femoral approach (differences n.s.), while those centers that were doing TRA in $>80 \%$ revealed a significant absolute difference between TRA and TFA of 5.2\% ( $\mathrm{p}=$ 0.00014 ) Their MACE for TRA was $10.3 \%$ vs. a non-acceptable $15,5 \%$ for TFA (Figure 1). In this low level TFA group the difference in NACE (major bleeding unrelated to coronary artery bypass surgery or major adverse cardiovascular events) between TRA and TFA was even more pronounced $(5,8 \%(\mathrm{p}=0.0001)$, unlike similar results for TRA and TFA in the two other groups. These finding are most likely related to atrophied transfemoral skills in the centers that by enlarge perform TRA, while those who cope with both techniques don't experience any significant difference of MACE and NACE between TRA and TFA [28].

The most recent randomized trial looking at mortality was the SAFARI STEMI trial. It included almost 2300 patients before the trial was stopped half way because of futility. There were no significant differences between patients assigned to radial and femoral access in the rates of reinfarction ( $1.8 \%$ vs. $1.6 \%$; RR, $1.07 ; 95 \% \mathrm{CI}, 0.57-2.00 ; \mathrm{P}$ $=0.83)$, stroke ( $1.0 \%$ vs. $0.4 \% ; \mathrm{RR}, 2.24 ; 95 \% \mathrm{CI}, 0.78-6.42 ; \mathrm{P}=0.12)$, and bleeding ( $1.4 \%$ vs. $2.0 \%$; RR, $0.71 ; 95 \% \mathrm{CI}, 0.38-1.33$; $\mathrm{P}=0.28$ ), survival or other clinical end points at 30 days after the use of radial access vs. femoral access in patients with STEMI referred for primary PCI [22]. 


\section{MACE Between TRA and TFA in the MATRIX Trial Stratified by Center's Proportion of Radial PCl}

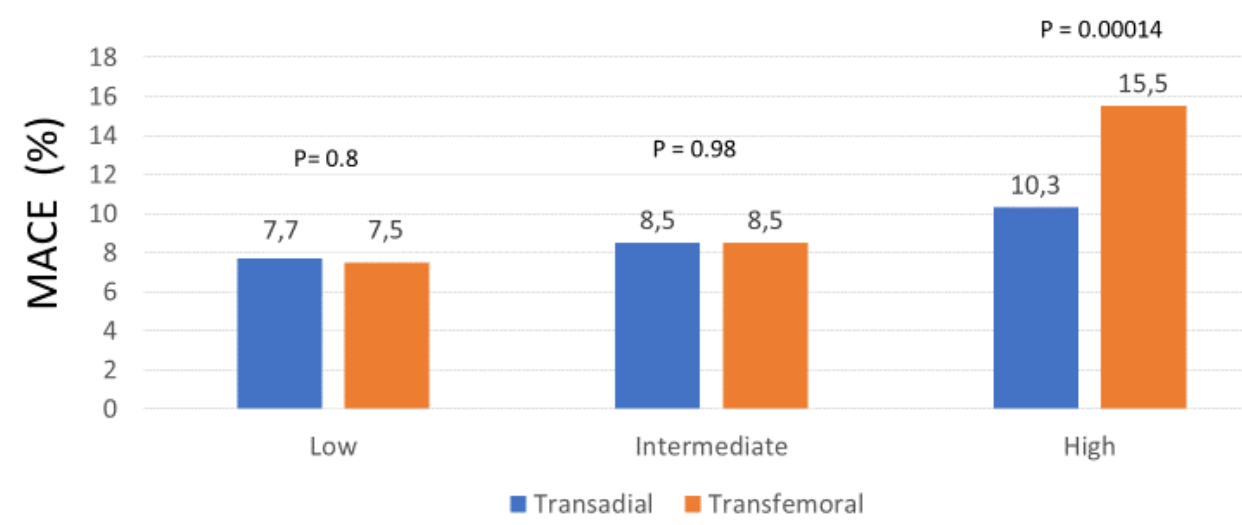

Figure 1: The MATRIX study divided patients into 3 groups based on the participating center's proportion of radial PCIs: "low" (14.9\% to $64.4 \%$ ), intermediate (65.4\% to $79.0 \%)$, and high (80.0\% to $98.0 \%)$. Only TFA by radial experts resulted in a significant increase in MACE [28].

\section{Metanalyses}

Metanalyses are often composed to overcome conflicting results of randomised trials. However, the selection process of which trials to include or exclude from analysis appears incomprehensible. In 2016 Ferrante included 24 studies that enrolled 22,843 PCI patients. Although the difference was small, radial was associated with significantly lower all-cause mortality and MACE, as well as less major bleeding and fewer vascular complications (Death of all cause: $-0.6 \%$ $(\mathrm{p}=0.001)$, MACE: $-1.1 \%(\mathrm{p}=0.002)$, Maj. Bleed: $-1 \%(\mathrm{p}<0.001)$, Maj. Vasc.Cpl.: $-0.9 \%(<0.001)[8]$. All randomised trials, including this metanalysis failed to mention any radial artery occlusion, a vascular complication that is not trivial and that occurs in up to $10 \%$ of the patients $[27,29]$.

Shah recently reported a metanalysis of 13 randomised trials of PCI in patients with ACS including 15,516 patients showing that following TFA the major bleeding, MACE and mortality rates of radial experts was significantly increased compared to their results of TRA and to those of transfemoral experts who had similar low complications rates for TFA and TRA. He concluded that the recently reported survival differences between TRA and TFA may have been driven by adverse events in the TFA groups, rather than by a beneficial effect of the TRA itself and that it is too early to label radial access a lifesaving procedure in invasively managed patients with ACS [27]. We do have a different explanation: To our view the inferior results of TFA of radial experts is probably attributed to insufficient skills in TFA (Figure 2).

\section{Image Quality and Interventional Success Rates}

After getting familiar with radial approach there is no reason to believe that it is not applicable for $>90 \%$ of diagnostic and interventional procedures. That being said, it is frequently more difficult to cannulate one or another coronary artery from a transradial approach. This invariably leads to poor quality diagnostic studies [13] and/or imperfect or failed PCI $[9,12]$. Transfemoral PCI is easier than radial PC because of fewer access problems and a higher likelihood of being able to use larger guides (e.g. $7 \mathrm{~F}$ and $8 \mathrm{~F}$ ) that might be necessary to enhance support and apply devices in parallel especially with complex lesion morphology. A small randomised trial $(\mathrm{n}=1024)$ showed a highly significantly increased success rate for TFA (99.8\% versus $96.5 \%$, p $<0.0001)$ following diagnostic and interventional coronary procedures [30].

\section{MACE of radial experts (RE) and non-radial experts (NRE) for transadial (TRA) and transfemoral (TRA) for PCl in ACS}

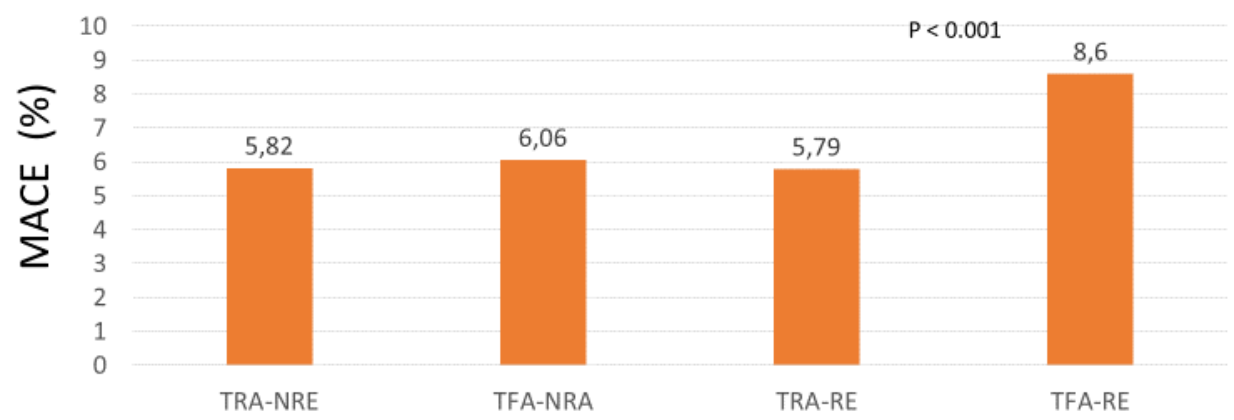

Figure 2: Meta-analysis of randomized controlled trials (Data from 13 trials including 15,615 patients). Only TFA performed by radial experts resulted in a significant increase in MACE [27]. 
A higher trans radial failure rate is also reflected by the large randomized trials: In the RIVAL trial that randomly investigated 7021 ACS patients with PCI, crossover related to failure of initial strategy was $7.0 \%$ in the radial group and $0.9 \%$ in the femoral group. Main reasons for failure were spasm (5\%), looped radial artery (1.3\%) and tortuous subclavian artery (1.9\%) [9]. RIFLE-STEACS reported a cross over rate from radial to femoral in 9.6\% [31]. MATRIX, the largest randomised comparison ( $\mathrm{n}=8404$ patients with ACS) reports a conversion to femoral of 5.8\% [12]. Switching to transfemoral upon failed trans radial PCI, will result in a $>90 \%$ success rate [25]. It can be assumed that significant time was wasted first trying TRA then crossing over to TFA. This is especially a problem in ACS patients.

\section{Radiation Exposure}

As long as procedure time is similar, radiation exposure to patients should not differ substantially between TRA and TFA. In the RIVAL trial, however, fluoroscopy times were significantly longer for the radial approach [32]. Due to a closer position and thus increased scattering, even highly experienced operators, receive at least twice the amount of radiation as do their femoral counterparts [14,33,34]. Therefore, compelling young operators to stick mainly with transradial approach, as put into practice in an increasing number of institutions, appears irresponsible unless the benefit for patients is crystal clear which is not the case until today.

\section{Patient Comfort}

Most operators agree that transradial approach is more convenient for the patient, mainly because early ambulation is possible in almost all patients, and also because postinterventional compression of the femoral puncture site of up to 6 hours can be distressing. On the other hand, different from femoral access that is generally tolerated very well, transradial access may be painful especially via small arteries and when spasm occurs. Diagnostic procedures via femoral artery may in $>>50 \%$ of the patients be performed with $4 \mathrm{~F}$ catheters (our practise since 1997), necessitating compression of the puncture side of $1 \mathrm{~h}$ only, and, following interventions with $6-8 \mathrm{~F}$ guides, we regularly apply closure devices that limit the (moderate) femoral compression to 2 hours and that also allow to ambulate the patients the same day. Since then we very rarely experience complaints about pain or discomfort.

\section{Conclusion}

The current movement from TFA to TRA in interventional treatment of ACS is not justified by evidence, since the randomised trials are showing conflicting results. If performed properly, TFA does not cause more complications than TRA but requires overnight hospitalisation unless vascular closure devices are applied. It is not justifiable to require operators to endure twice the radiation exposure to achieve an earlier ambulation. Forthcoming comparative studies need to include access- and sheath-removal protocols and need to appreciate postinterventional radial artery occlusion as a vascular complication.

\section{Appendix}

Recommended techniques for femoral artery puncture and groin management during and after diagnostic and therapeutic procedures performed via transfemoral approach.

\section{Puncturing the Femoral Artery}

Puncturing the femoral artery is not a technique that should be left at discretion to the operator but should follow strict rules: The needle should be razor-sharp to avoid arterial compression upon entry. For diagnostic procedures a $4 \mathrm{~F}$ sheath is sufficient in at least $80 \%$ of patients, with $5 \mathrm{~F}$ adequate for the rest. The target is the mid common femoral artery, a point that is usually positioned at the level of the centre of the femoral head. Higher or lower entries increase the rate of bleeding complications. At skin-level the entry is $1-2 \mathrm{~cm}$ below the inguinal ligament and the angle 30-45 degrees. Index and middle finger of the left hand (if puncture is performed from the right side) are firmly placed $3-5 \mathrm{~cm}$ proximally to feel the pulse and entry direction. Occasionally, if pulse is weak, fluoroscopy is needed to identify the correct direction. The "through-and through technique" should be strictly avoided. The wire should be advanced without resistance and the sheath inserted with a slight rotational motion and maintained pressure with the left hand proximally to avoid blood exit into the tissue.

\section{Sheath Removal and Compression}

The sheath should be removed immediately on the table or on a stretcher in the Cath lab to benefit from elastic recoil of the puncture, either by the operator or at least an experienced, well trained person. ACT should by $<200$ seconds (we rarely apply Protamine if ACT is still $>250 \mathrm{sec}$ ) and the systolic blood pressure should be $<160 \mathrm{mmHg}$. Sheath removal on the ward moreover by an inexperienced individual is a particularly bad idea. In patients at higher risk for bleeding we apply a closure device (Angioseal ${ }^{\bullet}$ St. Jude Medical - US/Terumo Europe).

Manual compression requires specific training and patience as well as a second person for assistance or replacement. The 3 middle fingers are firmly compressed $3-4 \mathrm{~cm}$ proximal to the skin puncture. The pressure should be enough to prevent any visible bleeding and any swelling. After 3-5 min the pressure may be released gradually to allow distal circulation and clotting. A frequent mistake resulting in inferior bleeding control, often used by beginners or petite persons, is to compress the site with the ball of the thumb or the balled fist. In prolonged compressions ( $>20 \mathrm{~min}$ ) we use an external pressure device (Femostop ${ }^{\circ}$ St. Jude Medical). A pressure dressing (e.g. Safeguard ${ }^{\circ}$ ) is applied if after 3-5 min of stopped manual compression there is no sign of bleeding or swelling.

The pressure dressing may be removed after 1 hour following $4 \mathrm{~F}$ catheterization, after 2 hours following $5 \mathrm{~F}$ and 6 hours with $6-8 \mathrm{~F}$. We do recommend closing devices in all suitable patients following $6 \mathrm{~F}$ and larger sheaths. The compression may be removed in these patients after 2 hours, and ambulation may be allowed in ordinary patients after 4 hours. We prefer to use a closure device (Angioseal ${ }^{\circ}$ ) in all patients that had 5-8 F sheaths, after an angiographic confirmation that the punctured common femoral artery is not severely stenosed and at least $6 \mathrm{~mm}$ in diameter. These patients don't need any compression and a dressing at moderate pressure for 2 hours. 


\section{References}

1. Neumann FJ, Miguel Sousa-Uva, Anders Ahlsson, Fernando Alfonso, Adrian P Banning, et al. (2018) 2018 ESC/EACTS Guidelines on myocardial revascularization. Eur Heart J 40: 87-165. [crossref]

2. Kolkailah AA, Rabah S Alreshq, Ahmed M Muhammed, Mohamed E Zahran, Marwah Anas El-Wegoud, et al. (2018) Transradial versus transfemoral approach for diagnostic coronary angiography and percutaneous coronary intervention in people with coronary artery disease. Cochrane Database Syst Rev 4: CD012318. [crossref]

3. Mason PJ, Binita Shah, Jacqueline E Tamis-Holland, John A Bittl, Mauricio G Cohen, et al. (2018) An Update on Radial Artery Access and Best Practices for Transradial Coronary Angiography and Intervention in Acute Coronary Syndrome: A Scientific Statement From the American Heart Association. Circ Cardiovasc Interv 11: e000035. [crossref]

4. Reifart J, Albrecht A, Haerer W, Levenson B, Ringwald G, et al. (2020) Acceptance and safety of femoral vs. radial access for percutaneous coronary intervention(PCI): Results from a large monitor-controlled German registry (QuIK). Submitted for publication.

5. Roffi M, Carlo Patrono, Jean-Philippe Collet, Christian Mueller, Marco Valgimigli, et al. (2016) 2015 ESC Guidelines for the management of acute coronary syndromes in patients presenting without persistent ST-segment elevation: Task Force for the Management of Acute Coronary Syndromes in Patients Presenting without Persistent ST-Segment Elevation of the European Society of Cardiology (ESC). Eur Heart J 37: 267-315. [crossref]

6. Ibanez B, Stefan James, Stefan Agewall, Manuel J Antunes, Chiara Bucciarelli-Ducci, et al. (2018) 2017 ESC Guidelines for the management of acute myocardial infarction in patients presenting with ST-segment elevation: The Task Force for the management of acute myocardial infarction in patients presenting with ST-segment elevation of the European Society of Cardiology (ESC). Eur Heart J 39: 119-177. [crossref]

7. Valgimigli M, et al. Transradial versus transfemoral intervention for acute myocardial infarction: a propensity score-adjusted and -matched analysis from the REAL (REgistro regionale AngiopLastiche dell'Emilia-Romagna) multicenter registry. JACC Cardiovasc Interv 5: 23-35.

8. Ferrante G, Sunil V Rao, Peter Jüni, Bruno R Da Costa, Bernhard Reimers, et al. (2016) Radial Versus Femoral Access for Coronary Interventions Across the Entire Spectrum of Patients With Coronary Artery Disease: A Meta-Analysis of Randomized Trials. JACC Cardiovasc Interv 9: 1419-1434. [crossref]

9. Jolly SS, Salim Yusuf, John Cairns, Kari Niemelä, Denis Xavier, et al. (2011) Radial versus femoral access for coronary angiography and intervention in patients with acute coronary syndromes (RIVAL): a randomised, parallel group, multicentre trial. Lancet 377: 1409-1420. [crossref]

10. Hvelplund A, Raban Jeger, Remo Osterwalder, Majken Bredahl, Jan Kyst Madsen, et al. (2011) The Angio-Seal femoral closure device allows immediate ambulation after coronary angiography and percutaneous coronary intervention. EuroIntervention 7: 234-241. [crossref]

11. Safley DM, Amit P Amin, John A House, Dmitri Baklanov, Rebecca Mills, et al. (2013) Comparison of costs between transradial and transfemoral percutaneous coronary intervention: a cohort analysis from the Premier research database. Am Heart J 165: 303-309. [crossref]

12. Valgimigli M, Andrea Gagnor, Paolo Calabró, Enrico Frigoli, Sergio Leonardi, et al. (2015) Radial versus femoral access in patients with acute coronary syndromes undergoing invasive management: a randomised multicentre trial. Lancet 385: 24652476. [crossref]

13. Chow WWK, Rong Bing, Juliana Kanawati, Jerrett Lau, Javed Sheriff, et al. (2018) A Comparison of Image Quality Using Radial vs Femoral Approaches in Patients Undergoing Diagnostic Coronary Angiography. I Invasive Cardiol 30: 411-415. [crossref]

14. Brasselet C, Thierry Blanpain, Sophie Tassan-Mangina, Alain Deschildre, Sébastien Duval, et al. (2008) Comparison of operator radiation exposure with optimized radiation protection devices during coronary angiograms and ad hoc percutaneous coronary interventions by radial and femoral routes. Eur Heart J 29: 63-70. [crossref]

15. Rashid M, Chun Shing Kwok, Samir Pancholy, Sanjay Chugh, Sasko A Kedev, et al. (2016) Radial Artery Occlusion After Transradial Interventions: A Systematic Review and Meta-Analysis. J Am Heart Assoc 5: e002686. [crossref]

16. Hahalis G, Konstantinos Aznaouridis, Gregory Tsigkas, Periklis Davlouros, Ioanna
Xanthopoulou, et al. (2017) Radial Artery and Ulnar Artery Occlusions Following Coronary Procedures and the Impact of Anticoagulation: ARTEMIS (Radial and Ulnar ARTEry Occlusion Meta-AnalysIS) Systematic Review and Meta-Analysis. J Am Heart Assoc 6: e005430. [crossref]

17. Gaudino M, Alessandro Leone, Andrea Lupascu, Amelia Toesca, Andrea Mazza, et al. (2015) Morphological and functional consequences of transradial coronary angiography on the radial artery: implications for its use as a bypass conduit. Eur J Cardiothorac Surg 48: 370-374. [crossref]

18. Kwok CS, Muhammad A Khan, Sunil V Rao, Tim Kinnaird, et al. (2015) Access and non-access site bleeding after percutaneous coronary intervention and risk of subsequent mortality and major adverse cardiovascular events: systematic review and meta-analysis. Circ Cardiovasc Interv 8: e001645. [crossref]

19. Kugelmass AD, David J Cohen, Phillip P Brown, April W Simon, Edmund R Becker et al. (2006) Hospital resources consumed in treating complications associated with percutaneous coronary interventions. Am J Cardiol 97: 322-327. [crossref]

20. Applegate RJ, Matthew T Sacrinty, Michael A Kutcher, Frederic R Kahl, Sanjay K Gandhi, et al. (2008) Trends in vascular complications after diagnostic cardiac catheterization and percutaneous coronary intervention via the femoral artery, 1998 to 2007. JACC Cardiovasc Interv 1: 317-326. [crossref]

21. Reifart J, Albrecht A, Haerer W, Levenson B, Ringwald G, et al. (2016) Femoral vs. radial access for coronary intervention (PCI) in patients with acute myocardial infarction (NSTEMI an STEMI): results from a large monitor-controlled German registry (QuIK), EuroPCR, Editor. EAPCI: Eurointerv.

22. Le May M, George Wells, Derek So, Aun Yeong Chong, Alexander Dick, et al. (2020) Safety and Efficacy of Femoral Access vs Radial Access in ST-Segment Elevation Myocardial Infarction: The SAFARI-STEMI Randomized Clinical Trial. JAMA Cardiol 5: 126-134. [crossref]

23. Arora N, Michael E Matheny, Carrie Sepke, Frederic S Resnic (2007) A propensity analysis of the risk of vascular complications after cardiac catheterization procedures with the use of vascular closure devices. Am Heart J 153: 606-611. [crossref]

24. Rashid M, Chun Shing Kwok, Samir Pancholy, Sanjay Chugh, Sasko A Kedev, et al (2016) Radial Artery Occlusion After Transradial Interventions: A Systematic Review and Meta-Analysis. J Am Heart Assoc. 5: e002686. [crossref]

25. Bertrand OF, Sunil V Rao, Samir Pancholy, Sanjit S Jolly, Josep Rodés-Cabau, et al (2010) Transradial approach for coronary angiography and interventions: results of the first international transradial practice survey. JACC Cardiovasc Interv 3: 1022 1031. [crossref]

26. Peter J Mason BS, Jaqueline E Tamis-Holland, John A Bittl, Mauricio G Cohen, Jordan Safirstein, et al. (2018) An Update on Radial Artery Access and Best Practices for Transradial Coronary Angiography and INtervention in Acute Coronary Syndrome. Circulation: Cardiovascular Interventions 11: e000035. [crossref]

27. Shah R, AR, Haji SA, Rashid A (2017) Mortality and operator experience with vascular access for percutaneous coronary intervention in patients with acute coronary syndromes: A pairwise and network meta-analysis of randomized controlled trials. Int J Cardiol 248: 114-119. [crossref]

28. Le May MR, K Singh, GA Wells (2015) Efficacy of Radial Versus Femoral Access in the Acute Coronary Syndrome: Is it the Operator or the Operation That Matters? JACC Cardiovasc Interv 8: 1405-1409. [crossref]

29. Zankl AR, M Andrassy, C Volz, B Ivandic, U Krumsdorf, et al. (2010) Radial artery thrombosis following transradial coronary angiography: incidence and rationale for treatment of symptomatic patients with low-molecular-weight heparins. Clin Res Cardiol 99: 841-847. [crossref]

30. Brueck M, Dirk Bandorski, Wilfried Kramer, Marcus Wieczorek, Reinhard Höltgen, et al. (2009) A randomized comparison of transradial versus transfemoral approach for coronary angiography and angioplasty. JACC Cardiovasc Interv 2: 1047-1054. [crossref]

31. Romagnoli E, Giuseppe Biondi-Zoccai, Alessandro Sciahbasi, Luigi Politi, Stefano Rigattieri, et al. (2012) Radial versus femoral randomized investigation in STsegment elevation acute coronary syndrome: the RIFLE-STEACS (Radial Versus Femoral Randomized Investigation in ST-Elevation Acute Coronary Syndrome) study. J Am Coll Cardiol 60: 2481-2489. [crossref]

32. Jolly SS, John Cairns, Kari Niemela, Philippe Gabriel Steg, Madhu K Natarajan, et al. (2013) Effect of radial versus femoral access on radiation dose and the importance of procedural volume: a substudy of the multicenter randomized RIVAL trial. JACC Cardiovasc Interv 6: 258-266. [crossref] 
Nicolaus Reifart (2020) Radial as the Default Approach: Are We Overexaggerating?

33. Lange HW, $\mathrm{H}$ von Boetticher (2012) Reduction of operator radiation dose by a pelvic lead shield during cardiac catheterization by radial access: comparison with femoral access. JACC Cardiovasc Interv 5: 445-449. [crossref]
34. Sciahbasi A, Christian Pristipino, Giuseppe Ambrosio, Isabella Sperduti, Enrico Vittorio Scabbia, et al. (2009) Arterial access-site-related outcomes of patients undergoing invasive coronary procedures for acute coronary syndromes (from the ComPaRison of Early Invasive and Conservative Treatment in Patients With NonST-ElevatiOn Acute Coronary Syndromes [PRESTO-ACS] Vascular Substudy). Am J Cardiol 103: 796-800. [crossref]

\section{Citation:}

Nicolaus Reifart (2020) Radial as the Default Approach: Are We Overexaggerating? Citation: Reifart N, Margolis J (2020) Radial as the Default Approach: Are We Overexaggerating? J Cardiol Clin Pract Volume 3(3): 1-6. 